\title{
Functional adaptations in the cecal and colonic metagenomes associated with the consumption of transglycosylated starch in a pig model
}

\author{
Barbara U. Metzler-Zebeli ${ }^{1 *}$, Monica A. Newman ${ }^{1}$, Dietmar Grüll² and Qendrim Zebeli ${ }^{1}$
}

\begin{abstract}
Background: Both phylogeny and functional capabilities within the gut microbiota populations are of great importance for influencing host health. As a novel type of resistant starch, transglycosylated starch (TGS) modifies the microbial community and metabolite profiles along the porcine gut, but little is known about the related functional adaptations in key metabolic pathways and their taxonomic identity.

Results: Metagenomic sequencing was used to characterize the functional alterations in the cecal and colonic microbiomes of growing pigs fed TGS or control starch (CON) diets for 10 days ( $n=8 /$ diet). Bacterial communities were clearly distinguishable at taxonomic and functional level based on the dietary starch, with effects being similar at both gut sites. Cecal and colonic samples from TGS-fed pigs were enriched in Prevotella, Bacteroides, Acidaminoccus and Veillonella, whereas Treponema, Ruminococcus, and Aeromonas declined at both gut sites compared to CON-fed pigs ( $\log _{2}$ fold change $> \pm 1 ; p<0.001(q<0.05)$ ). This was associated with increased enzymatic capacities for amino acid metabolism, galactose, fructose and mannose metabolism, pentose and glucuronate interconversions, citrate cycle and vitamin metabolism for samples from TGS-fed pigs. However, TGS-fed pigs comprised fewer reads for starch and sucrose metabolism and genetic information processing. Changes in key catabolic steps were found to be the result of changes in taxa associated with each type of starch. Functional analysis indicated steps in the breakdown of TGS by the action of $\alpha$ - and $\beta$-galactosidases, which mainly belonged to Bacteroides and Prevotella. Reads mapped to alphaamylase were less frequent in TGS- compared to CON-fed pigs, with the major source of this gene pool being Bacillus, Aeromonas and Streptococcus. Due to the taxonomic shifts, gene abundances of potent stimulants of the mucosal innate immune response were altered by the starches. The cecal and colonic metagenomes of TGS-fed pigs comprised more reads annotated in lipopolysaccharides biosynthesis, whereas they became depleted of genes for flagellar assembly compared to CON-fed pigs.

Conclusions: Metagenomic sequencing revealed distinct cecal and colonic bacterial communities in CON- and TGS-fed pigs, with strong discrimination among samples by functional capacities related to the respective starch in each pig's diet.
\end{abstract}

Keywords: Transglycosylated starch, Pig, Large intestine, Metagenome, Starch catabolism, Microbe-host-interaction

\footnotetext{
* Correspondence: barbara.metzler@vetmeduni.ac.at

${ }^{1}$ Department for Farm Animals and Veterinary Public Health, Institute of

Animal Nutrition and Functional Plant Compounds, University of Veterinary

Medicine Vienna, 1210 Vienna, Austria

Full list of author information is available at the end of the article
}

(c) The Author(s). 2019 Open Access This article is distributed under the terms of the Creative Commons Attribution 4.0 International License (http://creativecommons.org/licenses/by/4.0/), which permits unrestricted use, distribution, and

reproduction in any medium, provided you give appropriate credit to the original author(s) and the source, provide a link to the Creative Commons license, and indicate if changes were made. The Creative Commons Public Domain Dedication waiver (http://creativecommons.org/publicdomain/zero/1.0/) applies to the data made available in this article, unless otherwise stated. 


\section{Background}

The gut microbiota has a fundamental impact on host metabolism, immune functions and physiology with implications for gut and systemic health [1], whereby the diet of the host is their most important source of nutrients [2]. Poor nutrition will therefore not only impair the metabolic health of the host but lead to significant changes in the gut microbiota normobiosis [1]. Despite this awareness, our knowledge about the concurrent changes in the functional attributes in the bacterial microbiome is still limited.

Due to their high fermentability, there is growing interest to increase the proportion of resistant starch (RS) in human and animal diets for their potential prebiotic abilities. Resistant starches are long known for their blood glucose lowering effect because they resist digestion by mammalian enzymes in the small intestine [3]. Consequently, they become available to a large extent to the microbiota in the hindgut, altering the microbial composition and their metabolic activities including increased short-chain fatty acid (SCFA) production [46]. Currently, there are five types of RS of which type 2 and 3 are the best researched RS. In recent years, the development of low digestible chemically modified starches (RS type 4) has been promoted. These starches may fulfil a dual functionality by enhancing texture and rheological properties of processed foods [7] and, if low digestible, by increasing the daily dietary fiber intake. Especially, the modification of the chemical structure in chemically modified starches, such as cross-linking, esterification or tranglycosylation, may alter the pathways used by the microbes for the hydrolysis of the starch probably due to the promotion of other bacteria as with RS types 2 and $3[4,6,8,9]$.

Both phylogeny and functional capabilities within the gut microbiota populations are of great importance as they both impact host physiology and health [10]. Whereas progress has been made in regard to the knowledge of RS-related phylogenetic shifts, our current understanding of the functional adaptations in the gut microbiome due to RS consumption is still in its infancy. In-depth sequencing approaches using $16 \mathrm{~S}$ rRNA gene sequencing were extremely valuable to characterize the effect of the various RS types on the gastrointestinal bacterial microbiome $[6,8,9]$. Based on $16 \mathrm{~S}$ rRNA gene analysis, we could recently show for a transglycosylated starch (TGS) to largely alter the gastric, ileal, cecal and colonic bacterial microbiomes of growing pigs, with the qualitative changes being almost identical at the different gut sites [9]. Moreover, by identifying the most influential bacterial genera and medium-chain fatty acids and SCFA, the TGS induced a different bacterial signature on mucosal signaling compared to the waxy cornstarch fed as control [11]. The present objective was therefore to elucidate the changes in the predicted functional composition of the bacterial microbiome in the cecum and colon of growing pigs after TGS consumption using shotgun metagenome sequencing. We hypothesized that the alterations in the functional abilities that accompany the taxonomic changes in the cecum and colon of pigs will reveal the functional pathways required to utilize TGS and to interact with the host animal as part of the complex relationship between diet, microbial function and host physiology and metabolism.

\section{Results}

All pigs were clinically healthy throughout the experiment.

\section{General characteristics of the metagenomes}

To investigate alterations in the predicted functional composition of the bacterial microbiome in the cecum and colon of growing pigs after TGS consumption, DNA was extracted from intestinal samples of pigs fed either the TGS $(n=8)$ or control $(\mathrm{CON})$ diet $(n=8)$. Illumina NextSeq sequencing of multiplexed 150-bp libraries using a high-output, single-end protocol was employed to describe the taxonomic and functional microbial composition.

Shotgun metagenomic sequencing resulted in on average 13,206,140 and 14,094,761 reads in cecal and colonic samples, with a total of 1,973,401,005 and 2,105,585,724 bp, respectively, and an average read length of $149 \mathrm{bp}$ at both gut sites. After the quality control, 9,691,736 and $9,697,270$ proteins were predicted for cecal and colonic samples and 72.5 and $69.4 \%$ of the total reads in cecal and colonic samples were annotated as proteins functionally assigned, respectively.

\section{Comparison of taxonomic profiles reshaped by diet}

For the domain level, the microbiota profiles in the cecal and colonic samples generated by MG-RAST were predominated by Eubacteria, whereas archaeal counts only amounted to approximately $1 \%$ at both gut sites (Table 1). At phylum level, the cecal and colonic samples

Table 1 Taxonomic profile of cecal and colonic samples of CON- and TGS-fed pigs at domain level

\begin{tabular}{|c|c|c|c|c|c|}
\hline Domain & Mean $^{a}$ & $\log _{2}$ fold change & $\mathrm{SE}^{\mathrm{b}}$ & $p$ value & $q$ value $^{\ddagger}$ \\
\hline \multicolumn{6}{|l|}{ Cecum } \\
\hline Archaea & 24,714 & -0.018 & 0.067 & 0.789 & 0.930 \\
\hline Bacteria & $2,897,040$ & 0.729 & 0.206 & $<0.001$ & 0.003 \\
\hline \multicolumn{6}{|l|}{ Colon } \\
\hline Archaea & 34,107 & -0.128 & 0.076 & 0.091 & 0.135 \\
\hline Bacteria & 2,917,299 & 0.533 & 0.125 & $<0.001$ & $<0.001$ \\
\hline
\end{tabular}

${ }^{\ddagger}$ False discovery rate (Benjamini-Hochberg) corrected $p$ value

${ }^{a}$ Normalized reads (hit counts). Only the most abundant genera ( $>0.01 \%$ of all reads) that were altered by the dietary starch source are presented $(n=8$ per diet and gut site)

${ }^{\mathrm{b}}$ Standard error of the $\log _{2}$ fold change 
were dominated by Bacteroidetes, Firmicutes and Proteobacteria (Additional file 1: Figure S1). Within the archaeal community, Euryarchaeota $(\sim 95 \%)$ clearly predominated, followed by Crenaerchaeota $(\sim 4.0 \%)$ and Thaumaarchaeota $(\sim 0.5 \%)$ with similar abundances at both gut sites.

The TGS diet mainly shaped the cecal and colonic communities of the domain Bacteria (Table 1; Additional file 1: Figure S1 and S2), which increased by 0.73 and $0.53 \log _{2}$ folds in cecal and colonic digesta, respectively, of TGS-fed pigs compared to pigs receiving the control $(\mathrm{CON})$ diet $(p<0.05(q<0.01))$. In contrast, Archaea counts were similar between the feeding groups at both gut sites. In both gut segments (Additional file 1: Figure S1a and S1b), TGS-fed pigs comprised more Bacteroidetes and Actinobacteria, but less Spirochaetes counts compared to $\mathrm{CON}$-fed pigs $(p<0.05(q<0.10))$. With respect to archaeal phyla, only the Crenaerchaeota decreased in cecal samples of TGS- compared to CON-fed pigs by $-0.47 \log _{2}$ folds $(p=0.007 \quad(q=0.030))$. Dietary starch-related bacterial community profiles were shown in the distance matrices at phylum level (Additional file 1: Figure S1c and S1d). Of the predominant bacterial genera (Additional file 1: Figure $\mathrm{S} 2 \mathrm{a}$ and $\mathrm{S} 2 \mathrm{~b}$ ), the TGS diet enriched the cecal and colonic communities with Prevotella, Bacteroides, Acidaminococcus, Veillonella $(p<0.001 \quad(q<0.05))$ and Lactobacillus $(p<0.05$ $(q<0.1))$ compared to the CON diet. By contrast, pigs fed the TGS diet comprised less reads of the predominant genera Eubacterium, Treponema, Ruminococcus and Butyrivibrio in cecal samples as well as of Clostridium and Ruminococcus in colonic samples compared to CON-fed pigs $(p<0.05(q<0.1))$.

\section{Functional composition of the metagenome reshaped by} diet

To identify the major changes in the functional composition, we linked the genes in the present metagenomes to Kyoto Encyclopedia of Genes and Genomes (KEGG) orthology $(\mathrm{KO})$ classes and obtained the functional profiles of the metagenomes [12]. The functional profiles were first compared by non-parametric multidimensional scaling (NMDS), using reads that could be mapped to functional annotations (Fig. 1). The NMDS ordinal plots for $\mathrm{KO}$ level 3 and $\mathrm{KO}$ functions within 'carbohydrate metabolism' showed distinct clustering of the samples according to the two diets, whereas overlaps existed between gut sites (Fig. 1a and b). Nevertheless, when analyzing the single functions, the functional changes caused by the TGS product were qualitatively similar in cecal and colonic samples. Of the $62 \mathrm{KO}$ level 3 pathways that were differently abundant in the cecal samples, 31 pathways were enriched and 31 pathways were less frequent with the TGS compared to the CON diet (Table 2). In colonic digesta, $94 \mathrm{KO}$ level 3 pathways were differently abundant in TGS- and CON-fed pigs, 42 were more and 52 were less abundant with the TGS compared to the CON diet (Table 3). Differences between samples were detected for reads that were functionally annotated as being involved in 'amino acid metabolism,' 'carbohydrate metabolism,' 'biosynthesis of other secondary metabolites', 'glycan biosynthesis and metabolism,' 'membrane transport,' 'metabolism of cofactors and vitamins', and 'transport and catabolism' as well as related to the 'genetic information processing', irrespective of the gut site. Of interest, reads related to the citrate cycle and vitamin synthesis were more frequent, whereas genes related to transcription and translation
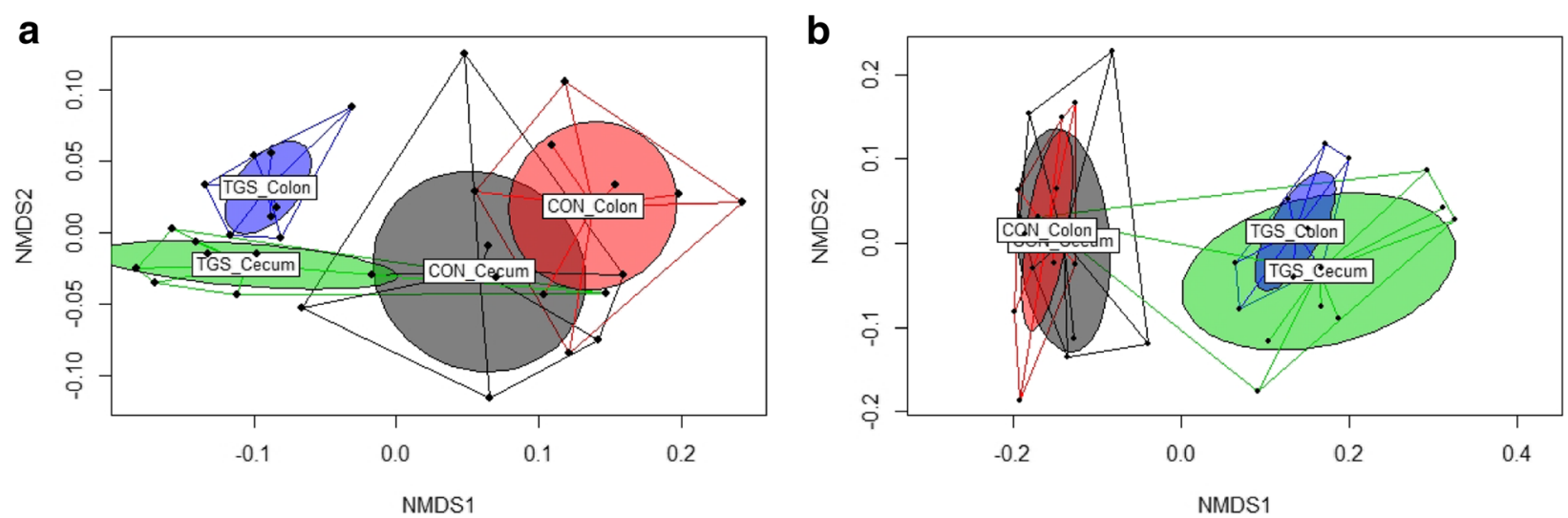

Fig. 1 Two-dimensional non-parametric multidimensional scaling (NMDS) ordination plots of predicted bacterial KEGG pathways (KEGG orthology (KO) level 3) and functions within the KEGG pathway 'carbohydrate metabolism' in cecal and colonic samples of control (CON) and transglycosylated starch (TGS)-fed pigs ( $n=8$ per diet and gut site). The NMDS plots were generated using Bray-Curtis distance metric between dietary starches. Each dot represents an individual samples; the circles indicate the SD. a KO level 3 pathways in cecal and colonic samples (stress $=0.1103$ ); and $\mathbf{b}$ 'carbohydrate metabolism'-related functions in cecal and colonic samples (stress $=0.0998$ ) 
Table 2 Selected KEGG pathways (KEGG orthology level 3) in cecal samples being differently enriched in CON- and TGS-fed pigs

\begin{tabular}{|c|c|c|c|c|c|}
\hline KEGG pathway & Mean $^{a}$ & $\log _{2}$ fold change & $\mathrm{SE}^{\mathrm{b}}$ & $p$ value & $q$ value \\
\hline 00633 Nitrotoluene degradation [PATH:ko00633] & 389 & 1.982 & 0.375 & $<0.001$ & $<0.001$ \\
\hline 00983 Drug metabolism - other enzymes [PATH:ko00983] & 1046 & 1.772 & 0.353 & $<0.001$ & $<0.001$ \\
\hline 00940 Phenylpropanoid biosynthesis [PATH:ko00940] & 9129 & 1.613 & 0.213 & $<0.001$ & $<0.001$ \\
\hline 02060 Phosphotransferase system (PTS) [PATH:ko02060] & 6289 & 1.040 & 0.217 & $<0.001$ & $<0.001$ \\
\hline 00130 Ubiquinone and other terpenoid-quinone biosynthesis [PATH:ko00130] & 3982 & 0.969 & 0.224 & $<0.001$ & $<0.001$ \\
\hline 00040 Pentose and glucuronate interconversions [PATH:ko00040] & 14,629 & 0.803 & 0.191 & $<0.001$ & $<0.001$ \\
\hline 04141 Protein processing in endoplasmic reticulum [PATH:ko04141] & 803 & 0.789 & 0.193 & $<0.001$ & $<0.001$ \\
\hline 00052 Galactose metabolism [PATH:ko00052] & 20,395 & 0.739 & 0.125 & $<0.001$ & $<0.001$ \\
\hline 00051 Fructose and mannose metabolism [PATH:ko00051] & 17,994 & 0.673 & 0.126 & $<0.001$ & $<0.001$ \\
\hline 00730 Thiamine metabolism [PATH:ko00730] & 9877 & 0.607 & 0.125 & $<0.001$ & $<0.001$ \\
\hline 02010 ABC transporters [PATH:ko02010] & 49,093 & -0.413 & 0.075 & $<0.001$ & $<0.001$ \\
\hline 03013 RNA transport [PATH:ko03013] & 1493 & -0.453 & 0.112 & $<0.001$ & $<0.001$ \\
\hline 04122 Sulfur relay system [PATH:ko04122] & 5609 & -0.976 & 0.197 & $<0.001$ & $<0.001$ \\
\hline 00360 Phenylalanine metabolism [PATH:ko00360] & 2011 & -1.286 & 0.311 & $<0.001$ & $<0.001$ \\
\hline 02040 Flagellar assembly [PATH:ko02040] & 7812 & -1.370 & 0.269 & $<0.001$ & $<0.001$ \\
\hline 00380 Tryptophan metabolism [PATH:ko00380] & 255 & -1.651 & 0.294 & $<0.001$ & $<0.001$ \\
\hline 00531 Glycosaminoglycan degradation [PATH:ko00531] & 391 & 1.077 & 0.275 & $<0.001$ & 0.001 \\
\hline 00860 Porphyrin and chlorophyll metabolism [PATH:ko00860] & 7948 & 0.594 & 0.152 & $<0.001$ & 0.001 \\
\hline 00680 Methane metabolism [PATH:ko00680] & 4678 & -0.375 & 0.096 & $<0.001$ & 0.001 \\
\hline 00630 Glyoxylate and dicarboxylate metabolism [PATH:ko00630] & 3354 & -0.418 & 0.108 & $<0.001$ & 0.001 \\
\hline 03070 Bacterial secretion system [PATH:ko03070] & 24,985 & -0.367 & 0.104 & $<0.001$ & 0.002 \\
\hline 00500 Starch and sucrose metabolism [PATH:ko00500] & 18,633 & -0.245 & 0.075 & 0.001 & 0.006 \\
\hline 04142 Lysosome [PATH:ko04142] & 3227 & 0.593 & 0.187 & 0.002 & 0.007 \\
\hline 00350 Tyrosine metabolism [PATH:ko00350] & 2333 & -0.932 & 0.294 & 0.002 & 0.007 \\
\hline 00511 Other glycan degradation [PATH:ko00511] & 2316 & 0.665 & 0.214 & 0.002 & 0.008 \\
\hline 04151 PI3K-Akt signaling pathway [PATH:ko04151] & 5164 & -0.822 & 0.267 & 0.002 & 0.009 \\
\hline 00590 Arachidonic acid metabolism [PATH:ko00590] & 829 & 1.026 & 0.351 & 0.003 & 0.014 \\
\hline 00300 Lysine biosynthesis [PATH:ko00300] & 23,314 & 0.136 & 0.047 & 0.004 & 0.014 \\
\hline $00510 \mathrm{~N}$-Glycan biosynthesis [PATH:ko00510] & 918 & 0.545 & 0.202 & 0.007 & 0.024 \\
\hline 00564 Glycerophospholipid metabolism [PATH:ko00564] & 5568 & 0.154 & 0.057 & 0.007 & 0.024 \\
\hline 00010 Glycolysis / Gluconeogenesis [PATH:ko00010] & 23,481 & -0.160 & 0.059 & 0.007 & 0.024 \\
\hline 00061 Fatty acid biosynthesis [PATH:ko00061] & 13,010 & -0.165 & 0.061 & 0.007 & 0.024 \\
\hline 00330 Arginine and proline metabolism [PATH:ko00330] & 26,554 & 0.284 & 0.106 & 0.007 & 0.025 \\
\hline 00071 Fatty acid metabolism [PATH:ko00071] & 133 & -1.077 & 0.423 & 0.011 & 0.036 \\
\hline 00900 Terpenoid backbone biosynthesis [PATH:ko00900] & 12,834 & 0.216 & 0.086 & 0.012 & 0.037 \\
\hline 00020 Citrate cycle (TCA cycle) [PATH:ko00020] & 15,173 & 0.415 & 0.171 & 0.015 & 0.045 \\
\hline 03020 RNA polymerase [PATH:ko03020] & 29,030 & -0.385 & 0.159 & 0.015 & 0.045 \\
\hline 02030 Bacterial chemotaxis [PATH:ko02030] & 8461 & -0.680 & 0.278 & 0.015 & 0.045 \\
\hline 03060 Protein export [PATH:ko03060] & 1992 & 0.193 & 0.083 & 0.020 & 0.056 \\
\hline 00520 Amino sugar and nucleotide sugar metabolism [PATH:ko00520] & 18,729 & 0.147 & 0.064 & 0.022 & 0.062 \\
\hline 00280 Valine leucine and isoleucine degradation [PATH:ko00280] & 5486 & -0.240 & 0.108 & 0.026 & 0.067 \\
\hline 00785 Lipoic acid metabolism [PATH:ko00785] & 167 & -0.642 & 0.287 & 0.025 & 0.067 \\
\hline 00030 Pentose phosphate pathway [PATH:ko00030] & 12,994 & -0.128 & 0.058 & 0.027 & 0.068 \\
\hline 00750 Vitamin B6 metabolism [PATH:ko00750] & 4284 & 0.280 & 0.131 & 0.033 & 0.077 \\
\hline
\end{tabular}


Table 2 Selected KEGG pathways (KEGG orthology level 3) in cecal samples being differently enriched in CON- and TGS-fed pigs (Continued)

\begin{tabular}{|c|c|c|c|c|c|}
\hline KEGG pathway & Mean $^{a}$ & $\log _{2}$ fold change & $\mathrm{SE}^{\mathrm{b}}$ & $p$ value & $q$ value $^{\ddagger}$ \\
\hline 00540 Lipopolysaccharide biosynthesis [PATH:ko00540] & 10,816 & 0.615 & 0.292 & 0.035 & 0.081 \\
\hline 00908 Zeatin biosynthesis [PATH:ko00908] & 2398 & 0.174 & 0.085 & 0.040 & 0.091 \\
\hline 04146 Peroxisome [PATH:ko04146] & 6168 & -0.179 & 0.088 & 0.041 & 0.091 \\
\hline 00053 Ascorbate and aldarate metabolism [PATH:ko00053] & 334 & 0.498 & 0.250 & 0.046 & 0.099 \\
\hline 03018 RNA degradation [PATH:ko03018] & 30,822 & -0.183 & 0.092 & 0.046 & 0.099 \\
\hline
\end{tabular}

${ }^{\ddagger}$ False discovery rate (Benjamini-Hochberg) corrected $p$ value

${ }^{a}$ Normalized reads (hit counts). Only the most abundant KEGG pathways ( $>0.01 \%$ of all reads) that were altered by the dietary starch source are presented ( $n=8$ per diet)

${ }^{\mathrm{b}}$ Standard error of the $\log _{2}$ fold change

declined in both cecal and colonic samples of TGScompared to CON-fed pigs. Also of note, reads annotated as methane metabolism and thus related to archaeal metabolism declined in cecal and colonic samples of TGS- compared to CON-fed pigs.

With respect to genes mapping for 'carbohydrate metabolism, read abundances for key catabolic steps in the degradation of glycans and energy metabolism were altered by the TGS compared to the CON diet (Additional file 1: Tables S1 and S2). Accordingly, cecal and colonic samples of TGS-fed pigs were enriched for genes mapping for 'galactose metabolism,' 'fructose and mannose metabolism, 'pentose and glucuronate interconversions' and 'citrate cycle', whereas the TGS decreased the enzymatic capacities related to 'starch and sucrose metabolism' compared to the CON diet at both gut sites. The bacterial contribution to the KO function 'starch and sucrose metabolism' largely changed with more hits being contributed by Prevotella, Bacteroides and Acidaminococcus in cecal and colonic samples of TGS-fed pigs (Fig. 2a and b). The screening of the functional enzymes indicated steps in the breakdown of TGS by the action of $\alpha$ - and $\beta$-galactosidases, fructokinase, galactokinase, arabinose isomerase and xylulokinase at both gut sites (Fig. 3a-f; Additional file 1: Tables S1-S6). Key enzymes that were mainly affected by the TGS diet within the 'starch and sucrose metabolism' included alpha-glucosidases and alpha-amylases. For instance, the reduced abundance of Bacillus, Aeromonas, Streptococcus, Butyrivibrio, Clostridium and Vibrio in TGS-compared to $\mathrm{CON}$-fed pigs reduced the reads related to the alpha-amylase gene amyA (Fig. $3 \mathrm{~g}$ and h; Additional file 1: Table S5).

Of note, the KO level 3 pathway 'lipopolysaccharide biosynthesis' was among the 10 most enriched pathways by the TGS diet at both gut sites (Fig. 4a and b; Additional file 1: Tables S1 and S2). These reads were again mostly contributed by the TGS-related increase in Prevotella, Acidaminococcus, Veillonella and Geobacter $(p<$ 0.05 ) and replaced the contribution of various Proteobacteria related to the $\mathrm{CON}$ diet in both cecal and colonic samples (Fig. 4a and b Additional file 1: Table S7).
Other functions related to potential virulence factors, such as genes mapping for 'flagellar assembly' were less frequent in TGS- compared to CON-fed pigs which could be associated with the reduced cecal and colonic abundance of Treponema, Aeromonas, Shewanella and Escherichia in TGS- compared to CON-fed pigs (Fig. 4c and d; Additional file 1: Table S8).

\section{Discussion}

While replacing rapidly digestible starch in the diet by RS may be a healthy option to control blood sugar and to increase the daily dietary fiber intake [3, 13], this commonly affects the gut microbial community with functional adaptations in key metabolic attributes which are indicated by alterations in metabolite profiles and microbial signaling at the host mucosa $[8,9,13]$. Previously, we studied the response of the gastrointestinal microbiome to RS type 4 (i.e. enzymatically modified starch (EMS) and TGS) based on 16S rRNA gene sequencing $[8,9]$ which provided valuable information on RS-related alterations in diversity and taxonomic composition and allowed us to predict the cecal functional metagenome content for the EMS from the 16S rRNA dataset. However, due to the inherent limitation that $16 \mathrm{~S}$ rRNA gene sequencing alone is insufficient to reveal microbial function, we used a whole genome shotgun metagenomics approach to characterize the bacterial functional adaptations that were associated with the taxonomic shifts caused by the TGS in the large intestine. The present shotgun metagenomic data demonstrate that replacing part of the dietary starch by TGS produced drastic and similarly directed shifts in taxonomy and functions in the cecal and colonic microbiota of growing pigs, despite the continuing changes in substrate availability from cecum to colon. In providing a deeper insight into the archaeal community than our previous 16S rRNA gene approach, results showed that TGS-related changes were mostly within Eubacteria, whereas only subtle changes in the archaeal community (i.e. Crenarchaeota) were detected. Since methanogens lack the enzymatic capacities to break down external 
Table 3 Selected KEGG pathways (KEGG orthology level 3) in colonic samples being differently enriched in CON- and TGS-fed pigs

\begin{tabular}{|c|c|c|c|c|c|}
\hline KEGG pathway & Mean $^{a}$ & $\log _{2}$ fold change & $S E^{b}$ & $p$ value & $q$ value $^{\ddagger}$ \\
\hline 00633 Nitrotoluene degradation [PATH:ko00633] & 661 & 2.575 & 0.351 & $<0.001$ & $<0.001$ \\
\hline 00983 Drug metabolism - other enzymes [PATH:ko00983] & 818 & 1.910 & 0.160 & $<0.001$ & $<0.001$ \\
\hline 00531 Glycosaminoglycan degradation [PATH:ko00531] & 397 & 1.868 & 0.276 & $<0.001$ & $<0.001$ \\
\hline 02060 Phosphotransferase system (PTS) [PATH:ko02060] & 9299 & 1.739 & 0.228 & $<0.001$ & $<0.001$ \\
\hline 00940 Phenylpropanoid biosynthesis [PATH:ko00940] & 8963 & 1.542 & 0.148 & $<0.001$ & $<0.001$ \\
\hline 00130 Ubiquinone and other terpenoid-quinone biosynthesis [PATH:ko00130] & 3636 & 1.510 & 0.145 & $<0.001$ & $<0.001$ \\
\hline 00710 Carbon fixation in photosynthetic organisms [PATH:ko00710] & 202 & 1.422 & 0.373 & $<0.001$ & $<0.001$ \\
\hline 00590 Arachidonic acid metabolism [PATH:ko00590] & 922 & 1.336 & 0.321 & $<0.001$ & $<0.001$ \\
\hline 00910 Nitrogen metabolism [PATH:ko00910] & 1675 & 1.152 & 0.262 & $<0.001$ & $<0.001$ \\
\hline 00740 Riboflavin metabolism [PATH:ko00740] & 4762 & 1.040 & 0.199 & $<0.001$ & $<0.001$ \\
\hline 00511 Other glycan degradation [PATH:ko00511] & 2787 & 1.016 & 0.174 & $<0.001$ & $<0.001$ \\
\hline 00051 Fructose and mannose metabolism [PATH:ko00051] & 16,391 & 0.746 & 0.089 & $<0.001$ & $<0.001$ \\
\hline 04141 Protein processing in endoplasmic reticulum [PATH:ko04141] & 784 & 0.728 & 0.143 & $<0.001$ & $<0.001$ \\
\hline 00052 Galactose metabolism [PATH:ko00052] & 21,809 & 0.713 & 0.091 & $<0.001$ & $<0.001$ \\
\hline 04142 Lysosome [PATH:ko04142] & 2980 & 0.683 & 0.153 & $<0.001$ & $<0.001$ \\
\hline 00860 Porphyrin and chlorophyll metabolism [PATH:ko00860] & 10,480 & 0.647 & 0.102 & $<0.001$ & $<0.001$ \\
\hline 00040 Pentose and glucuronate interconversions [PATH:ko00040] & 13,379 & 0.593 & 0.101 & $<0.001$ & $<0.001$ \\
\hline 00750 Vitamin B6 metabolism [PATH:ko00750] & 3823 & 0.559 & 0.127 & $<0.001$ & $<0.001$ \\
\hline 00281 Geraniol degradation [PATH:ko00281] & 614 & 0.549 & 0.144 & $<0.001$ & $<0.001$ \\
\hline 00020 Citrate cycle (TCA cycle) [PATH:ko00020] & 16,331 & 0.533 & 0.089 & $<0.001$ & $<0.001$ \\
\hline 00730 Thiamine metabolism [PATH:ko00730] & 8664 & 0.522 & 0.097 & $<0.001$ & $<0.001$ \\
\hline 00790 Folate biosynthesis [PATH:ko00790] & 3122 & 0.507 & 0.124 & $<0.001$ & $<0.001$ \\
\hline 00400 Phenylalanine tyrosine and tryptophan biosynthesis [PATH:ko00400] & 17,524 & 0.386 & 0.094 & $<0.001$ & $<0.001$ \\
\hline 00330 Arginine and proline metabolism [PATH:ko00330] & 27,975 & 0.296 & 0.058 & $<0.001$ & $<0.001$ \\
\hline 00340 Histidine metabolism [PATH:ko00340] & 15,847 & 0.252 & 0.048 & $<0.001$ & $<0.001$ \\
\hline 03018 RNA degradation [PATH:ko03018] & 31,058 & -0.206 & 0.052 & $<0.001$ & $<0.001$ \\
\hline 03010 Ribosome [PATH:ko03010] & 59,767 & -0.279 & 0.066 & $<0.001$ & $<0.001$ \\
\hline 00010 Glycolysis / Gluconeogenesis [PATH:ko00010] & 25,589 & -0.307 & 0.043 & $<0.001$ & $<0.001$ \\
\hline 00970 Aminoacyl-tRNA biosynthesis [PATH:ko00970] & 96,501 & -0.377 & 0.066 & $<0.001$ & $<0.001$ \\
\hline 03070 Bacterial secretion system [PATH:ko03070] & 26,474 & -0.429 & 0.079 & $<0.001$ & $<0.001$ \\
\hline 03020 RNA polymerase [PATH:ko03020] & 30,568 & -0.656 & 0.072 & $<0.001$ & $<0.001$ \\
\hline 00310 Lysine degradation [PATH:ko00310] & 788 & -0.991 & 0.220 & $<0.001$ & $<0.001$ \\
\hline 04151 PI3K-Akt signaling pathway [PATH:ko04151] & 4509 & -1.058 & 0.083 & $<0.001$ & $<0.001$ \\
\hline 00360 Phenylalanine metabolism [PATH:ko00360] & 2719 & -1.103 & 0.149 & $<0.001$ & $<0.001$ \\
\hline 04122 Sulfur relay system [PATH:ko04122] & 7389 & -1.137 & 0.086 & $<0.001$ & $<0.001$ \\
\hline 00350 Tyrosine metabolism [PATH:ko00350] & 3212 & -1.404 & 0.164 & $<0.001$ & $<0.001$ \\
\hline 00785 Lipoic acid metabolism [PATH:ko00785] & 187 & -1.562 & 0.244 & $<0.001$ & $<0.001$ \\
\hline 00380 Tryptophan metabolism [PATH:ko00380] & 304 & -1.595 & 0.181 & $<0.001$ & $<0.001$ \\
\hline 00791 Atrazine degradation [PATH:ko00791] & 196 & -2.087 & 0.374 & $<0.001$ & $<0.001$ \\
\hline 03040 Spliceosome [PATH:ko03040] & 203 & -2.108 & 0.292 & $<0.001$ & $<0.001$ \\
\hline 00660 C5-Branched dibasic acid metabolism [PATH:ko00660] & 142 & -4.390 & 0.563 & $<0.001$ & $<0.001$ \\
\hline 00540 Lipopolysaccharide biosynthesis [PATH:ko00540] & 9910 & 0.965 & 0.261 & 0.000 & 0.001 \\
\hline 02010 ABC transporters [PATH:ko02010] & 65,587 & -0.243 & 0.070 & 0.001 & 0.001 \\
\hline 00500 Starch and sucrose metabolism [PATH:ko00500] & 22,311 & -0.256 & 0.073 & $<0.001$ & 0.001 \\
\hline
\end{tabular}


Table 3 Selected KEGG pathways (KEGG orthology level 3) in colonic samples being differently enriched in CON- and TGS-fed pigs (Continued)

\begin{tabular}{|c|c|c|c|c|c|}
\hline KEGG pathway & Mean $^{a}$ & $\log _{2}$ fold change & $S E^{b}$ & $p$ value & $q$ value $^{\ddagger}$ \\
\hline 03420 Nucleotide excision repair [PATH:ko03420] & 23,037 & -0.270 & 0.078 & 0.001 & 0.001 \\
\hline 00680 Methane metabolism [PATH:ko00680] & 5869 & -0.359 & 0.099 & $<0.001$ & 0.001 \\
\hline 00071 Fatty acid metabolism [PATH:ko00071] & 136 & -1.551 & 0.426 & $<0.001$ & 0.001 \\
\hline 00230 Purine metabolism [PATH:ko00230] & 49,811 & -0.134 & 0.040 & 0.001 & 0.002 \\
\hline 00640 Propanoate metabolism [PATH:ko00640] & 530 & -0.546 & 0.165 & 0.001 & 0.002 \\
\hline 00510 N-Glycan biosynthesis [PATH:ko00510] & 785 & 0.460 & 0.148 & 0.002 & 0.004 \\
\hline 00900 Terpenoid backbone biosynthesis [PATH:ko00900] & 13,580 & 0.143 & 0.046 & 0.002 & 0.004 \\
\hline 00620 Pyruvate metabolism [PATH:ko00620] & 22,435 & -0.192 & 0.063 & 0.002 & 0.005 \\
\hline 03060 Protein export [PATH:ko03060] & 1837 & 0.318 & 0.107 & 0.003 & 0.006 \\
\hline 00250 Alanine aspartate and glutamate metabolism [PATH:ko00250] & 69,446 & 0.133 & 0.045 & 0.003 & 0.007 \\
\hline 00260 Glycine serine and threonine metabolism [PATH:ko00260] & 42,580 & -0.110 & 0.039 & 0.005 & 0.009 \\
\hline 03013 RNA transport [PATH:ko03013] & 1374 & -0.285 & 0.111 & 0.010 & 0.020 \\
\hline 00550 Peptidoglycan biosynthesis [PATH:ko00550] & 17,416 & -0.145 & 0.059 & 0.014 & 0.027 \\
\hline 01040 Biosynthesis of unsaturated fatty acids [PATH:ko01040] & 108 & -1.247 & 0.512 & 0.015 & 0.028 \\
\hline 00053 Ascorbate and aldarate metabolism [PATH:ko00053] & 466 & 0.595 & 0.250 & 0.017 & 0.031 \\
\hline 03030 DNA replication [PATH:ko03030] & 31,056 & -0.233 & 0.098 & 0.018 & 0.031 \\
\hline 00300 Lysine biosynthesis [PATH:ko00300] & 23,272 & 0.118 & 0.050 & 0.019 & 0.033 \\
\hline 00520 Amino sugar and nucleotide sugar metabolism [PATH:ko00520] & 20,592 & 0.107 & 0.046 & 0.020 & 0.035 \\
\hline 00362 Benzoate degradation [PATH:ko00362] & 479 & 0.797 & 0.368 & 0.030 & 0.051 \\
\hline 03440 Homologous recombination [PATH:ko03440] & 20,390 & -0.127 & 0.060 & 0.035 & 0.058 \\
\hline 00440 Phosphonate and phosphinate metabolism [PATH:ko00440] & 788 & 0.628 & 0.307 & 0.041 & 0.065 \\
\hline 00561 Glycerolipid metabolism [PATH:ko00561] & 5347 & 0.269 & 0.135 & 0.046 & 0.071 \\
\hline 00290 Valine leucine and isoleucine biosynthesis [PATH:ko00290] & 23,041 & 0.159 & 0.080 & 0.046 & 0.071 \\
\hline 00270 Cysteine and methionine metabolism [PATH:ko00270] & 33,967 & 0.118 & 0.061 & 0.055 & 0.083 \\
\hline 02030 Bacterial chemotaxis [PATH:ko02030] & 8408 & 0.622 & 0.327 & 0.057 & 0.085 \\
\hline 00030 Pentose phosphate pathway [PATH:ko00030] & 14,947 & -0.111 & 0.059 & 0.062 & 0.090 \\
\hline
\end{tabular}

${ }^{\ddagger}$ False discovery rate (Benjamini-Hochberg) corrected $p$ value

${ }^{a}$ Normalized reads (hit counts). Only the most abundant KEGG pathways ( $>0.01 \%$ of all reads) that were altered by the dietary starch source are presented ( $n=8$ per diet)

${ }^{\mathrm{b}}$ Standard error of the $\log _{2}$ fold change

glycans to monosaccharides, they rely on the provision of monosaccharides and hydrogen generated during the fermentation of carbohydrates [14, 15]. Consequently, the TGS-related reduced enzymatic capacities annotated in methane metabolism may indicate a lower substrate availability for methanogens in the hindgut of TGScompared to $\mathrm{CON}$-fed pigs.

In considering the nature of the alpha- and beta-glycosidic bonds in the TGS product, many of those linkages can be found in non-digestible oligo- and polysaccharides of plant, yeast and algae origin, privileging bacteria that are commonly associated with the breakdown of such oligo- and polysaccharides. While being a metabolically extremely versatile bacterial group [16], this likely explains the great contribution of Prevotella to the enrichment in genes for key catabolic steps. Similarly,
Bacteroides are adept at using both complex plant and host-derived glycans as the main sources of energy [17, 18], explaining their contribution to taxonomic hit counts and metagenome functions. TGS-related alterations in digesta viscosity, intestinal substrate flow and retention in the various intestinal segments are other possible modes of action how the TGS diet may have modified the enzymatic capacities in cecal and colonic digesta. An increased flow of dietary protein and peptides in TGS-fed pigs may have stimulated bacterial amino acid metabolism in the cecal and colonic regions which would be supported by the many related genes that were enriched with the TGS compared to the CON diet. Alternatively, as sufficient energy needs to be available [19], the greater flow of starch with the TGS diet to the cecum-colon region may have stimulated bacterial 


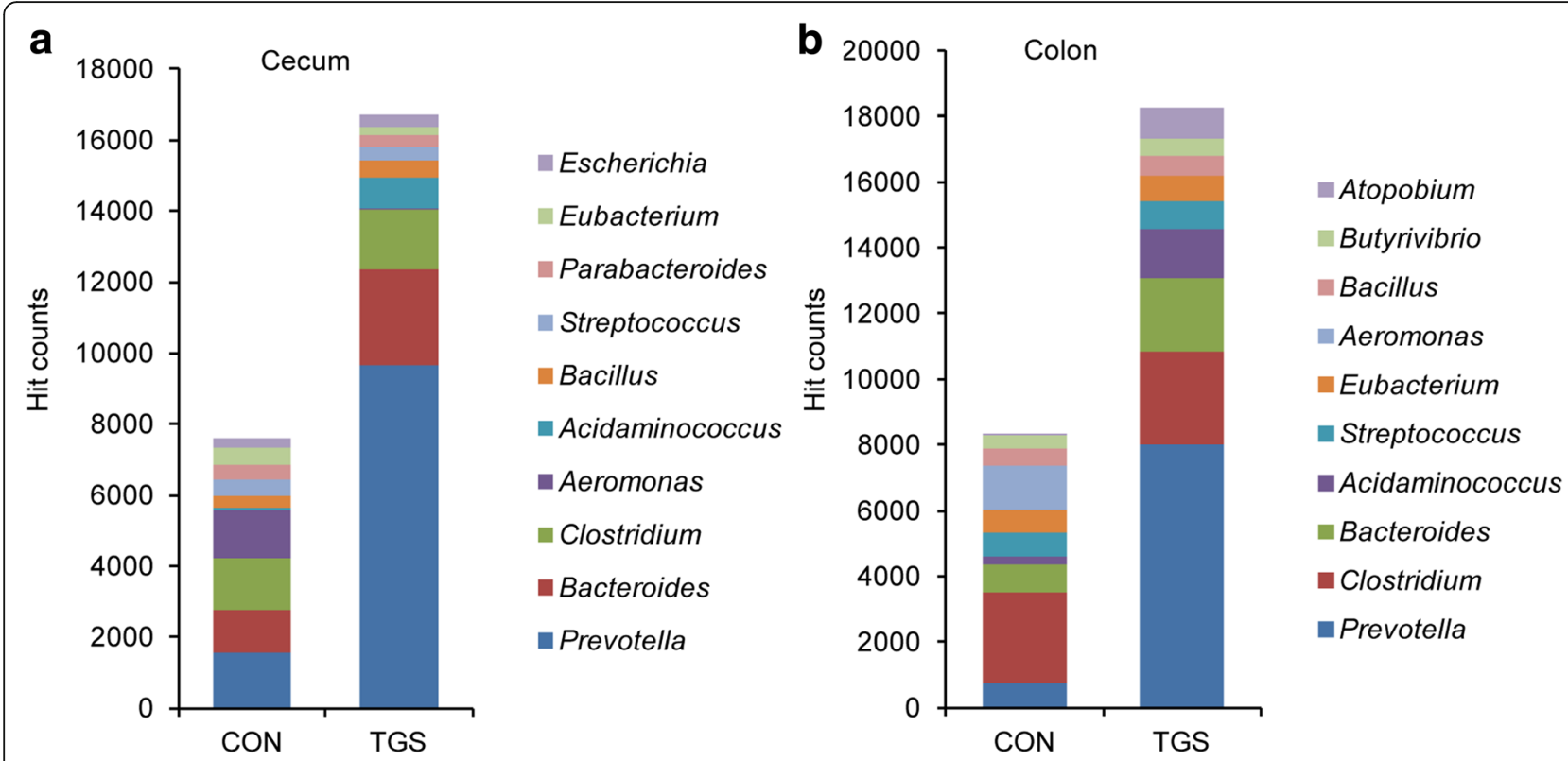

Fig. 2 Taxonomic identity of predicted functions within the KEGG pathway 'starch and sucrose metabolism' in cecal (a) and colonic samples (b) of control starch (CON)- and transglycosylated starch (TGS)-fed pigs ( $n=8$ per diet and gut site). Values are presented as the mean of hit counts per dietary starch. Hit counts were normalized with DESeq2-size factors. Only the taxonomic identity of the 10 most abundant genera is presented

amino acid synthesis, leading to the enrichment in the respective pathways. Against this background, the drastic increase in Acidaminococcus, for instance, may be more related to greater substrate flow to the cecum and colon and availability of amino acids as their main carbon source (e.g. glutamate) [20] in TGS-fed pigs than to the TGS itself. Other taxa, such as Eubacterium and Veillonella, may have relied on cross-feeding of primary fermentation metabolites such as lactate and succinate for the formation of propionate and butyrate, respectively [21]. This clearly implies that the different metabolic pathways used for the breakdown of the two dietary starches resulted in the production of different primary metabolites which then modulated the abundance of taxa relying on them. This assumption is supported by the increase in succinyl-CoA synthetase [22] and decrease in acetate and butyrate kinases [21] as well as by our findings for enhanced cecal and colonic lactate and propionate concentrations with the TGS diet, respectively [9].

The NMDS ordination plots showed strong differences in the predicted bacterial functions, supporting that the TGS required a different set of alpha- and beta-glucanases to deconstruct the additional linkages produced during transglycosylation compared to the waxy cornstarch. Although the TGS diet only decreased the hit count abundance of the KEGG pathway 'starch and sucrose metabolism' by about $0.25 \log _{2}$ fold in both cecal and colonic samples, the catabolic abilities within this pathway as well as the origin of those genes largely differed. Since the taxonomic and functional prediction is based on the alignment of short $150 \mathrm{bp}$ fragments, care should be taken in the interpretation of the obtained origin of the predicted functions. The decline in the predicted alpha-amylases and maltases was assignable to the decrease in Bacillus, Aeromonas, Streptococcus and Clostridium with the TGS compared to the CON diet. Enzymes within the KEGG pathways 'galactose metabolism' and 'fructose and mannose metabolism' may have been useful in the breakdown of TGS, especially enzymes predicted to encode beta-galactosidases. This enzyme capacity is involved in the hydrolysis of beta-glycosidic bonds in beta-galactosides, including lactose [11,23], and has been reported to decrease in the intestinal metagenome of pigs after weaning due to the weaning-related decrease in $\mathrm{Bac}$ teroides and increase in Prevotella [24]. Compared to the weaning shift, the predicted three-time greater contribution of Prevotella to the beta-galactosidase genes indicated a growth advantage for this genus in pigs fed the TGS diet compared to Bacteroides. Moreover, two scenarios may explain the enrichment of the TGS-metagenomes with alpha-galactosidase (galA), with the effect being stronger in colonic than in cecal samples. Alpha-galactosidase ( galA) capacities are involved in the degradation of glycoproteins such as mucins [25], which may have played a greater role as bacterial substrate in the colon compared to the cecum in TGS-fed pigs. Alternatively, bacterial alpha-galactosidase activity breaks down melibiose [26], 

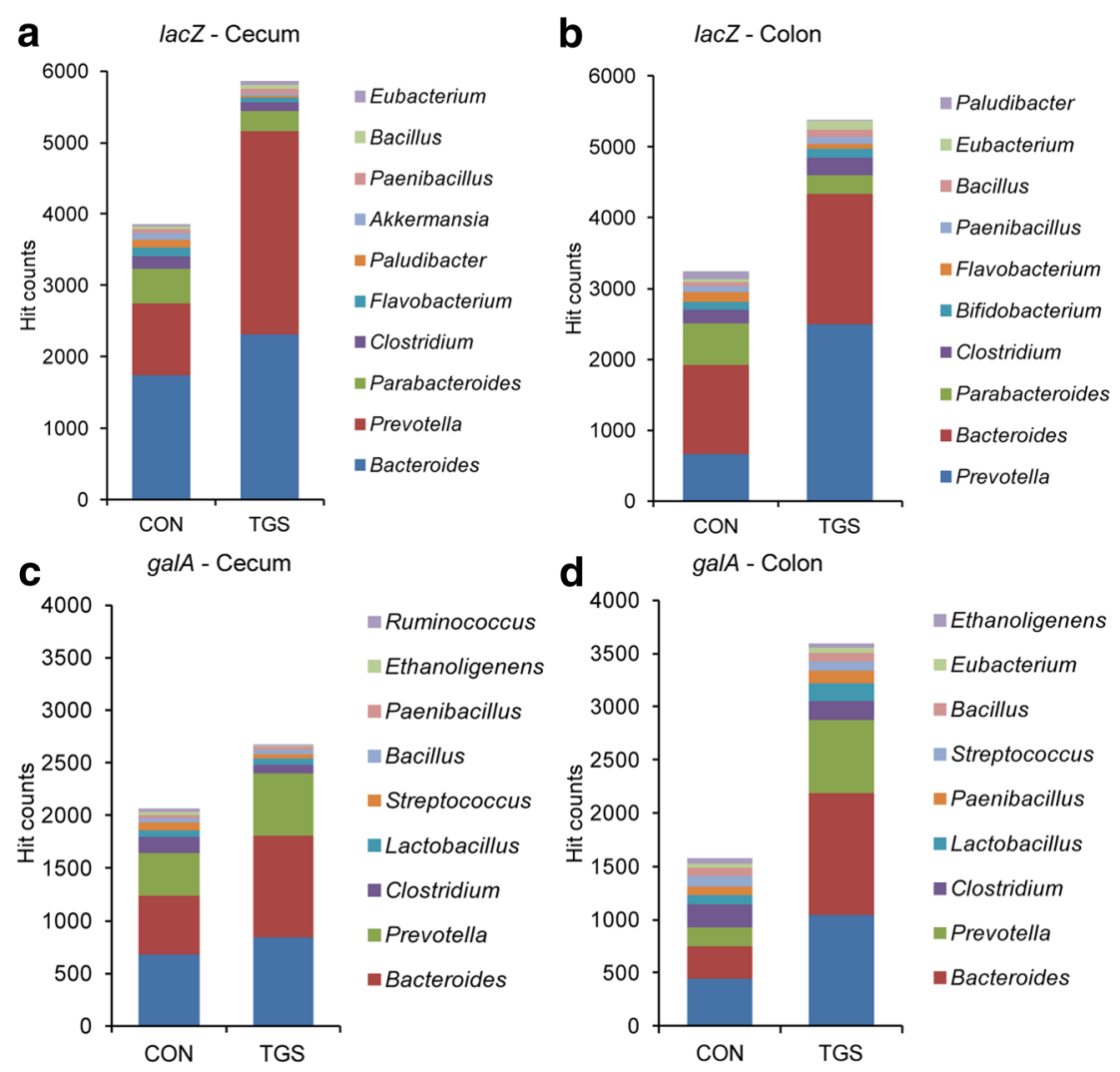

e
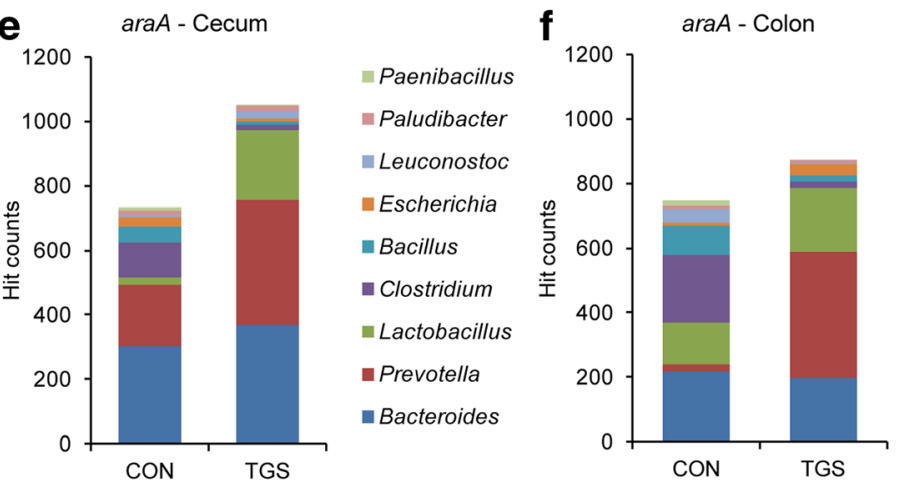

Vibrio

- Paludibacter

Escherichia

Leuconostoc

nacillus

- Clostridium

- Prevotella

- Lactobacillus

- Bacteroides

g

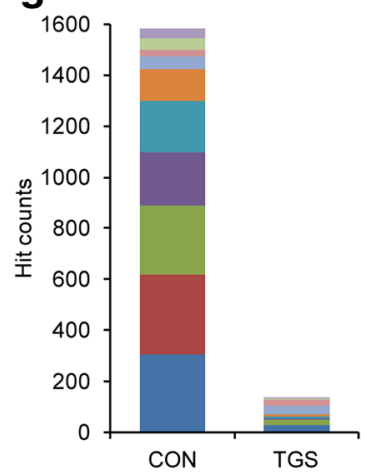

h

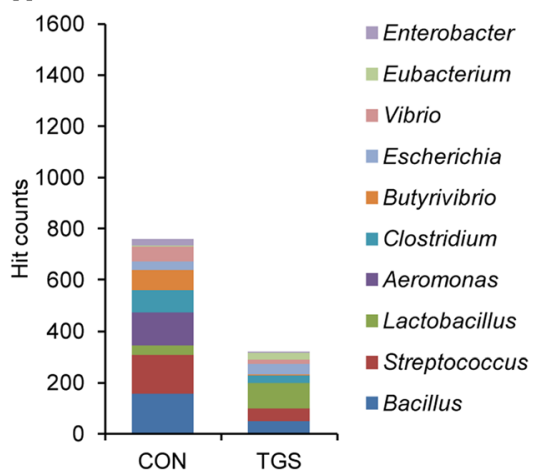

Fig. 3 (See legend on next page.) 
(See figure on previous page.)

Fig. 3 Taxonomic identity of selected predicted functions related to carbohydrate metabolism in cecal and colonic samples of control starch (CON)- and transglycosylated starch (TGS)-fed pigs ( $n=8$ per diet and gut site). $\mathbf{a}$ beta-galactosidase (lacZ) in cecal samples; $\mathbf{b}$ beta-galactosidase (lacZ) in colonic samples; c alpha-galactosidase (galA) in cecal samples; $\mathbf{d}$ alpha-galactosidase (galA) in colonic samples; e alpha-amylase (amyA) in cecal samples; $\mathbf{f}$ alpha-amylase ( $a m y A)$ in colonic samples; $\mathbf{g} \mathrm{L}$-arabinose isomerase $(\operatorname{araA})$ in cecal samples; and $\mathbf{h} \mathrm{L}$-arabinose isomerase (araA) in colonic samples. Values are presented as the mean of hit counts per dietary starch. Hit counts were normalized with DESeq2-size factors. Only the taxonomic identity of the most abundant genera is presented. Abundance change significance $(p<0.05$ (false discovery rate, $q<0.10)$ ) between dietary starches is presented in Additional file 1: Table S3-S6

the chiral form of lactose. Therefore, the enhanced predicted alpha-galactosidase activity in colonic digesta of TGS-fed pigs may have been also in relation to a greater flow of slowly fermentable TGS fragments to the colonic region. In line with this assumption, the TGS-associated enrichment in the predicted KEGG pathway 'citrate cycle' supports enhanced fermentative activity and bacterial energy acquisition in the cecum and colon of TGS- compared to CON-fed pigs. Moreover, the metabolic pathways feeding intermediates into this pathway differed between diets. In CON-fed pigs, glycolysis and pyruvate metabolism probably provided the necessary precursors [15], whereas in TGS-fed pigs the predicted 'pentose and glucuronate interconversions' pathway may have played a greater role in transferring metabolites from the TGS-enriched KEGG pathways 'galactose metabolism' and
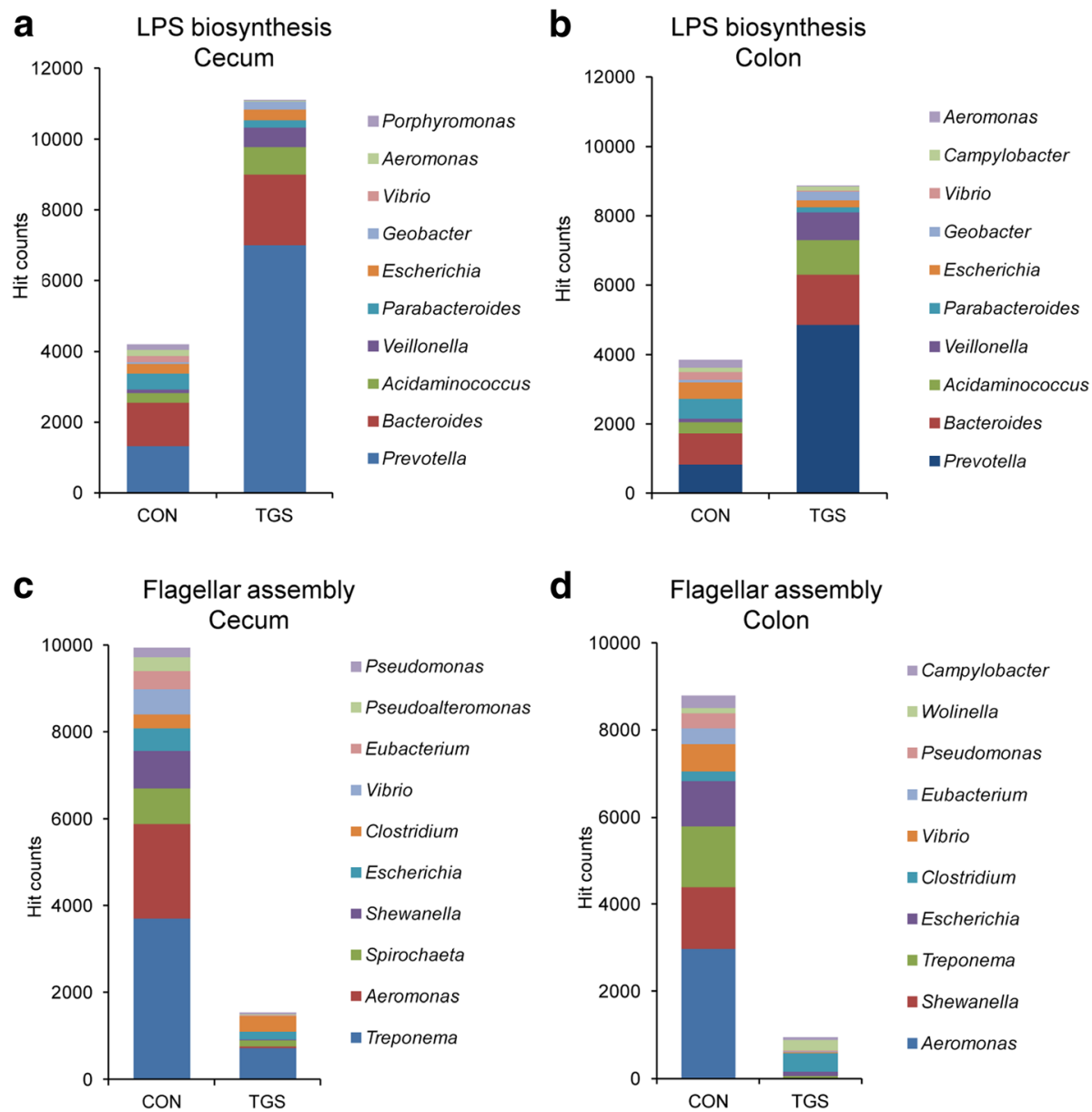

Fig. 4 Taxonomic identity of predicted functions for lipopolysaccharide biosynthesis and flagellar assembly in cecal and colonic samples of control starch (CON) and transglycosylated starch (TGS)-fed pigs ( $n=8$ per diet and gut site). a gene abundance related to lipopolysaccharide biosynthesis in cecal samples; $\mathbf{b}$ gene abundance related to lipopolysaccharide biosynthesis in colonic samples; $\mathbf{c}$ gene abundance related to flagellar assembly in cecal samples; $\mathbf{d}$ gene abundance related to flagellar assembly in colonic samples. Values are presented as the mean of hit counts per dietary starch. Hit counts were normalized with DESeq2-size factors. Only the taxonomic identity of the 10 most abundant genera is presented. Abundance change significance $(p<0.05$ (false discovery rate, $q<0.10)$ ) between dietary starches is presented in Table S7 and S8 
'fructose and mannose metabolism' to provide precursors for the 'citrate cycle', 'amino sugar and nucleotide sugar metabolism' [15], thereby stimulating the biosynthesis of B-vitamins and ascorbate. Despite the less efficient absorption of vitamins from the large intestinal segments, an enhanced bacterial vitamin synthesis may still benefit the host animal $[27,28]$. While those predicted genes were enriched, predicted DNA replication, transcription and translation processes were repressed in cecal and colonic metagenomes of TGS- compared to CON-fed pigs, potentially indicating bacterial metabolic priorities at the time point of sampling.

Besides utilizing diet- and host-related substrates, the intestinal microbiota serve as a rich source of immune-reactive molecules $[29,30]$. Due to the taxonomic enrichment with Gram-negative bacteria, especially Prevotella, Acidaminococcus, and Veillonella, the cecal and colonic metagenomes of TGS-fed pigs comprised more reads predicted to encode proteins for the biosynthesis of lipopolysaccharides; a potent stimulant of the mucosal innate immune response by binding to Toll-like receptor 4 (TLR4) [31]. As the fatty acid composition of the lipid A component of lipopolysaccharides is responsible for the activation of the TLR4 response and differs among the various Gram-negative bacteria [32], the decrease in the TLR4 expression that we observed for the cecal and colonic mucosa [11] may be directly related to the taxonomic identity of those reads as well as to the development of a certain immune tolerance [30]. For instance, the predicted contribution of Prevotella to the lipopolysaccharides biosynthesis genes increased from about $50 \%$ in $\mathrm{CON}$-fed pigs to more than $80 \%$ in TGS-fed pigs, lowering the predicted contribution of Proteobacteria whose lipopolysaccharides commonly provoke a strong immune response [33]. With the associated decrease in Proteobacteria, e.g. Aeromonas and Vibrio, the metagenomes of TGS-fed pigs also became depleted with other genes predicted to code for potential virulence factors, such as 'flagellar assembly', which play a significant role in enhancing the pathogen's capability to cause disease [34]. This may have contributed to the lower expression of genes related to the innate immune response in TGS-fed pigs as well, which we reported in our companion article [11]. These findings for the TGS show the importance of the provenience of microbial immune stimulants and emphasizes the necessity to discover their bacterial origin in relation to dietary changes in human and animal studies.

\section{Conclusion}

The present shotgun metagenomics approach demonstrated that replacing part of the dietary starch by TGS produced similarly taxonomic and functional shifts in the microbial community with some variations due to substrate availability in the cecum-colon region of growing pigs. Samples showed strong discrimination to the respective starch in pig's diet by altered functional capacities for amino acid, carbohydrate, and energy metabolism, synthesis of vitamins and virulence factors, as well as cellular information processing. Key enzymatic capacities for the breakdown of TGS may be found within the $\mathrm{KO}$ pathways 'galactose metabolism' and 'fructose and mannose metabolism'. Present results also emphasize that changes in the abundance and provenience of microbial immune stimulants necessitate to discover their bacterial origin in relation to dietary changes and to assess potential consequences for host physiology, metabolism and health. Nevertheless, it needs to be considered that the present shotgun metagenomic approach based on multiplexing of 150-bp DNA libraries can only provide a prediction of the bacterial metabolic capacities and should be complemented by metatranscriptomics and metaproteomics in the future.

\section{Methods \\ Animal experiment}

The experimental design and diets of this study have been described in the sister article [9], presenting data from the same pigs as used in this study. In brief, 16 growing male Large White pigs (age: 4 months; initial BW: $45.4 \pm 4.2 \mathrm{~kg}$ ) from 6 litters were obtained from the University research farm (University of Veterinary Medicine Vienna, Vienna, Austria) and randomly assigned to 1 of 2 diets in a randomized design with 2 replicate batches, with 4 animals per treatment in each batch [9]. Pigs were individually fed and housed in metabolism pens $(1.20 \mathrm{~m} \times 1.00 \mathrm{~m})$ with Plexiglas walls to allow visual contact. Each cage was equipped with one nipple drinker with free access to demineralized water, one feeder and one heating lamp.

\section{Diets and feeding}

The two semi-purified diets consisted of purified cornstarch, casein, lignocellulose (FibreCell M1; agromed Austria $\mathrm{GmbH}$, Kremsmünster, Austria), rapeseed oil, vitamins, and minerals and were identical except for the starch component [9] and were formulated to meet or exceed current recommendations for nutrient requirements for growing pigs [35]. The CON diet comprised a rapidly digestible waxy cornstarch (Agrana Research and Innovation Center GmbH (ARIC), Tulln, Austria), whereas $50 \%$ of the native waxy cornstarch was replaced by the TGS product (ARIC) in the TGS diet [9]. The TGS was produced via an acid-catalyzed transglycosylation of the native waxy cornstarch, which rearranges the glycosidic bonds. As a result, the TGS had 8 types of glycosydic bonds (i.e. $\alpha(1,2), \alpha(1,3), \alpha(1,4), \alpha(1,6), \beta(1,2)$, $\beta(1,3), \beta(1,4)$, and $\beta(1,6)$-glycosidic bonds) and a total 
dietary fiber content of 50\% (method 2009.01) [36], whereas the native waxy cornstarch had only $\alpha(1,4)$ - and $\alpha(1,6)$-glycosidic bonds.

Pigs were manually fed the experimental diets three times daily $(0800,1100$ and $1600 \mathrm{~h})$ to mimic human meal patterns, whereby feed allowances were calculated to surpass pig's appetite [9]. Feed spillage and residuals in feeding bowls were collected after feeding. Diets were analyzed for dry matter, crude protein, calcium, phosphorus and starch as previously described $[9,11]$.

\section{Collection of intestinal digesta samples}

Intestinal digesta samples were collected $2 \mathrm{~h}$ after morning feeding. After sedation (Narketan, $10 \mathrm{ml} / \mathrm{kg}$ body weight; Ketamine $\mathrm{HCl}$; Vétoquinol AG, Ittigen, Austria; and Stresnil, $3 \mathrm{ml} / \mathrm{kg}$ body weight; Azaperone; Biokema SA, Crissier, Switzerland), pigs were euthanized by intracardiac injection of T61 (10 ml/kg, Embutramide; MSD Animal Health, Vienna, Austria) [9]. The abdominal cavity was opened. The whole gastrointestinal tract was removed from the abdomen, and the small and large intestines were identified, isolated, and carefully dissected them from the mesentery. The cecum and mid colon (top of the beehive) were identified, opened at the mesentery and emptied. The luminal digesta was collected, thoroughly homogenized and stored on ice until long-term storage at $-80^{\circ} \mathrm{C}$.

\section{DNA isolation and shotgun metagenomic sequencing}

Total DNA was isolated from approximately $250 \mathrm{mg}$ of cecal, and mid-colonic digesta using the PowerSoil DNA isolation kit (MoBio Laboratories, Carlsbad, CA) according to the manufacturer's instructions except an additional heating step at $70^{\circ} \mathrm{C}$ for $10 \mathrm{~min}$ between mixing the digesta samples with $\mathrm{C} 1$ buffer and bead beating to ensure proper lysis of bacteria $[9,37]$. The DNA concentration was measured with a Qubit 2.0 fluorometer (Life Technologies, Carlsbad, CA) using the Qubit doublestranded DNA HS assay kit (Life Technologies, Carlsbad, CA). DNA isolates were sent to Microsynth (Balgach, Switzerland) for shotgun metagenome sequencing using the Illumina NextSeq 500 v2 sequencing platform (Illumina Inc., San Diego, CA).Total genomic DNA was prepared using the Illumina TruSeq Reagent Chemistry and the Illumina TruSeq nano protocol for whole genome shotgun sequencing of multiplexed 150-bp libraries by Microsynth AG (Balgach, Switzerland) using a high-output, single-end protocol. Barcoded sample libraries were sequenced and FASTQ files were de-multiplexed, quality filtered, and trimmed of Illumina adaptor residuals to 150 bp by Microsynth AG, yielding an average of 12-17 million reads per sample.

\section{Bioinformatic processing of sequences}

The taxonomic and functional profiles were built by processing and annotating the quality-filtered DNA sequences using the MG-RAST (Metagenomics Rapid Annotation using Subsystem Technology, v4.0; Agronne National Laboratories; https://metagenomics.anl.gov/) pipeline [38-40]. After removal of host genomic DNA, duplicate reads and $16 \mathrm{~S}$ rRNA reads, putative protein coding features were predicted using GragGeneScan [41] and clustered at $90 \%$ identity. Protein similarity search against the M5NR protein database was done with sBLAT [42]. Cecal and colonic samples from the same pig were treated as separate samples and processed independently. The Kyoto Encyclopedia of Genes and Genomes (KEGG) [43, 44] and KEGG orthology (KO) annotation source of MG-RAST were used for taxonomic and functional analysis of cecal and colonic samples. Subsets of the reads annotated in a KO class for taxonomic identification were created in MG-RAST. The criteria applied for inclusion were a maximum e-value cutoff of 1 e- 05 , a minimum identity of $60 \%$ and a minimum alignment length of 15 .

\section{Statistical analysis}

A power test analysis estimated as described in Metzler-Zebeli et al. [11] based on recent data for intestinal microbiome composition [9] using the SAS software (version 9.4; SAS Inst. Inc., Cary, NC, USA) was performed to identify the number of observations needed for the present pig experiment. The power test analysis indicated that a statistical power of more than $90 \%$ for a sample size of $n=8$ and $\alpha=0.05$ could be expected, enabling sufficient power to reject the null-hypothesis $(\mathrm{H} 0)$, if $\mathrm{H} 0$ was false $(p=1-\beta)$.

The "DESeq" function within the DESeq2 package (version 1.14.1) [45] in $\mathrm{R}$ was used to test for differentially abundant taxa and putative functions by dietary starch, at each taxonomic and functional level. Data were normalized to the size factors of the libraries and dispersion estimation and were listed as normalized read counts per feature. This function models raw counts using a negative binomial distribution and adjusts internally for "size factors" which normalize for differences in sequencing depth between sample libraries. In addition, we pre-filtered the taxonomic and functional datasets to keep only features that have at least 10 reads total using the $\mathrm{R}$ command in DESeq2 "rowSums(counts(deseq_data)) $\geq 10$ " to remove low-count taxa and functions for the phylum through species level analysis as well as KO level 3 and $\mathrm{KO}$ function analysis.

DEseq2 default settings were used to replace and filter for count outliers. Differential taxa and function abundance between treatments were identified using the "Wald" test [45]. Data were listed as normalized read 
counts per feature. The correction of $p$ values relating to the taxonomic and functional profiles were performed using the Benjamini-Hochberg false discovery rate (FDR) [46]. To account for the multiple comparisons at each taxonomic and functional level, we considered a type I error rate of $\leq 0.05$ and a FDR-adjusted $p$ value ( $q$ value) $\leq 0.10$ as significant. Mean counts for each dietary starch were computed using the "sapply" function in DESeq2. Log-transformed DESeq2-normalized data were used to create heatmaps and to compute distance matrices (Euclidian distance) for bacterial phyla and genera and functional attributes (KO level 3) using the pheatmap $R$ package in R. Statistical assessment of dissimilarity matrices (Bray-Curtis) from the functional data was done using NMDS with the "metaMDS" function in the vegan $R$ package (version 2.5.1) [47]. All statistical tests were carried out using $\mathrm{R}$ studio (version 1.0.136).

\section{Additional file}

Additional file 1: Table S1. Selected KEGG orthology functions within the KEGG pathway 'carbohydrate metabolism' in cecal samples being differently enriched in CON- and TGS-fed pigs. Table S2. Selected KEGG orthology functions within the KEGG pathway 'carbohydrate metabolism' in colonic samples being differently enriched in CON- and TGS-fed pigs. Table S3. Taxonomic identity of lacZ genes being differently enriched in cecal and colonic samples of CON- and TGS-fed pigs. Table S4.

Taxonomic identity of galA genes being differently enriched in cecal and colonic samples of CON- and TGS-fed pigs. Table S5. Taxonomic identity of amyA genes being differently enriched in cecal and colonic samples of CON- and TGS-fed pigs. Table S6. Taxonomic identity of araA genes being differently enriched in cecal and colonic samples of CON- and TGS-fed pigs. Table S7. Taxonomic identity of genes predicted to encode proteins within the KEGG pathway 'lipopolysaccharide biosynthesis' differently enriched in cecal and colonic samples of CON- and TGS-fed pigs.

Table S8. Taxonomic identity of genes predicted to encode proteins within the KEGG pathway 'flagellar assembly' differently enriched in cecal and colonic samples of CON- and TGS-fed pigs. Figure S1. Heatmaps of abundances (hit counts) and distance matrices (Euclidian distance) of cecal and colonic samples at phylum level in control starch (CON)- and transglycosylated starch (TGS)-fed pigs (P1-P16). a phyla abundances in cecum; b phyla abundances in colon; c distance matrix of cecal samples; and d distance matrix of colonic samples. Hit counts were normalized with DESeq2-size factors. Abundance change significance between dietary starches is indicated by * $(p<0.05$ (false discovery rate, $q<0.10)$ ). Figure S2. Heatmaps of differently abundant genera (hit counts) in of cecal (a) and colonic samples (b) from control starch (CON)- and transglycosylated starch (TGS)-fed pigs (P1-P16). Only the 30 most abundant genera that were differently affected by the dietary starch source are presented. Hit counts were normalized with DESeq2-size factors. Abundance change significance between dietary starches is $p<0.05$ (false discovery rate, $q<0.10)$ ). (PDF $286 \mathrm{~kb}$ ) (PDF $250 \mathrm{~kb}$ )

\section{Abbreviations}

CON: Control starch; KEGG: Kyoto Encyclopedia of Genes and Genomes; KO: KEGG orthology; MG-RAST: Metagenomics Rapid Annotation using Subsystem Technology; NMDS: Non-parametric multidimensional scaling; RS: Resistant starch; SCFA: Short-chain fatty acids; TGS: Transglycosylated starch

\section{Acknowledgements}

The authors thank G. Kvapil, C. Grabner, N. Schneider and A. Sener (Institute of Animal Nutrition and Functional Plant Compounds) for assistance in the animal trial and laboratory analyses. T. Molnar-Wegerth is thanked for assistance with starch provision and analysis (Agrana Research \& Innovation Center $\mathrm{GmbH}$ ) as well as Agromed Austria GmbH (Kremsmünster, Austria) for providing FibroCell M1.

\section{Funding}

This project was funded by the Austrian Research Promotion Agency (FFG), BRIDGE project (No. 836447-"Healthy Carbohydrates"). The funders did not have any role in the design of the study, collection, analysis and interpretation of the data, decision to publish, and in writing the manuscript.

\section{Availability of data and materials}

The data are publicly available on the MG-RAST server (project ID mgp21210).

\section{Author's contributions}

BM-Z, QZ, and DG conceived and designed the experiments. BM-Z and MN performed the experiments and lab work. BM-Z analyzed and interpreted the data. BM-Z drafted the manuscript. QZ and MN revised the manuscript. $B M-Z$ has the primary responsibility for the final content. All of the authors read and approved the final manuscript.

\section{Ethics approval and consent to participate}

All procedures involving animal handling and treatment were approved by the institutional ethics committee of the University of Veterinary Medicine and the national authority according to paragraph 26 of Law for Animal Experiments, Tierversuchsgesetz - TVG (GZ 68.205/0063-WF/II3b/2014).

\section{Consent for publication}

Not applicable.

\section{Competing interests}

Agrana Research \& Innovation Center $\mathrm{GmbH}$ provided support in the form of salary for author DG, but did not have any additional role in the study design, data collection and analysis, decision to publish, or preparation of the manuscript.

\section{Publisher's Note}

Springer Nature remains neutral with regard to jurisdictional claims in published maps and institutional affiliations.

\section{Author details}

${ }^{1}$ Department for Farm Animals and Veterinary Public Health, Institute of Animal Nutrition and Functional Plant Compounds, University of Veterinary Medicine Vienna, 1210 Vienna, Austria. ${ }^{2}$ Agrana Research \& Innovation Center $\mathrm{GmbH}, 3430$ Tulln, Austria.

Received: 13 September 2018 Accepted: 22 April 2019

Published online: 02 May 2019

\section{References}

1. Tremaroli $V$, Bäckhed F. Functional interactions between the gut microbiota and host metabolism. Nature. 2012;489(7415):242-9. https://doi.org/10.1038/ nature 11552.

2. Ley RE, Turnbaugh PJ, Klein S, ecology GJIM. Human gut microbes associated with obesity. Nature. 2006;444(7122):1022-3.

3. Birt DF, Boylston T, Hendrich S, Jane JL, Hollis J, Li L, McClelland J, Moore S, Phillips GJ, Rowling M, Schalinske K, Scott MP, Whitley EM. Resistant starch: promise for improving human health. Adv Nutr. 2013;4(6):587-601. https:// doi.org/10.3945/an.113.004325.

4. Haenen D, Souza da Silva C, Zhang J, Koopmans SJ, Bosch G, Vervoort J, Gerrits WJ, Kemp B, Smidt H, Müller M, Hooiveld GJ. Resistant starch induces catabolic but suppresses immune and cell division pathways and changes the microbiome in the proximal colon of male pigs. J Nutr. 2013;143(12): 1889-98. https://doi.org/10.3945/jn.113.182154.

5. Sun Y, Zhou L, Fang L, Su Y, Zhu W. Responses in colonic microbial community and gene expression of pigs to a long-term high resistant starch diet. Front Microbiol. 2015;6:877. https://doi.org/10.3389/fmicb.2015. 00877.

6. Sun Y, Su Y, Zhu W. Microbiome-metabolome responses in the cecum and colon of pig to a high resistant starch diet. Front Microbiol. 2016;7:779. https://doi.org/10.3389/fmicb.2016.00779. 
7. Singh J, Dartois A, Kaur L. Starch digestibility in food matrix: a review. Trends Food Sci Technol. 2010;21:168-80.

8. Metzler-Zebeli BU, Schmitz-Esser S, Mann E, Grüll D, Molnar T, Zebeli Q. Adaptation of the cecal bacterial microbiome of growing pigs in response to resistant starch type 4. Appl Environ Microbiol. 2015;81(24):8489-99. https://doi.org/10.1128/AEM.02756-15.

9. Newman MA, Petri RM, Grüll D, Zebeli Q, Metzler-Zebeli BU. Transglycosylated starch modulates the gut microbiome and expression of genes related to lipid synthesis in liver and adipose tissue of pigs. Front Microbiol. 2018;9:224. https://doi.org/10.3389/fmicb.2018.00224.

10. Singh KM, Shah TM, Reddy B, Deshpande S, Rank DN, Joshi CG. Taxonomic and gene-centric metagenomics of the fecal microbiome of low and high feed conversion ratio (FCR) broilers. J Appl Genetics. 2014;55:145-54. https:// doi.org/10.1007/s13353-013-0179-4.

11. Metzler-Zebeli BU, Newman MA, Grüll D, Zebeli Q. Consumption of transglycosylated starch down-regulates expression of mucosal innate immune response genes in the large intestine using a pig model. $\mathrm{Br}$ J Nutr. 2018;119:1366-77.

12. Jovel J, Patterson J, Wang W, Hotte N, O'Keefe S, Mitchel T, Perry T, Kao D, Mason AL, Madsen KL, Wong GK. Characterization of the gut microbiome using 16S or shotgun metagenomics. Front Microbiol. 2016;7:459. https:// doi.org/10.3389/fmicb.2016.00459.

13. Yang X, Darko KO, Huang Y, He C, Yang H, He S, Li J, Li J, Hocher B, Yin Y. Resistant starch regulates gut microbiota: structure, biochemistry and cell Signalling. Cell Physiol Biochem. 2017;42(1):306-18. https://doi.org/10.1159/ 000477386.

14. Nkamga VD, Henrissat B, Drancourt M. Archaea: essential inhabitants of the human digestive microbiota. Hum Microb J. 2016;3:1-8 https://doi.org/10. 1016/j.humic.2016.11.005

15. Macfarlane S, Macfarlane GT. Regulation of short-chain fatty acid production. Proc Nutr Soc. 2003;62(1):67-72.

16. Accetto T, Auguštin G. Polysaccharide utilization locus and CAZYme genome repertoires reveal diverse ecological adaptation of Prevotella species. Syst Appl Microbiol. 2015;38(7):453-61. https://doi.org/10.1016/j. syapm.2015.07.007.

17. Marcobal A, Barboza M, Sonnenburg ED, Pudlo N, Martens EC, Desai P, Lebrilla CB, Weimer BC, Mills DA, German JB, Sonnenburg JL. Bacteroides in the infant gut consume milk oligosaccharides via mucus-utilization pathways. Cell Host Microbe. 2011;10(5):507-14.

18. Arnal G, Cockburn DW, Brumer H, Koropatkin NM. Structural basis for the flexible recognition of a-glucan substrates by Bacteroides thetaiotaomicron SusG. Protein Sci. 2018;30. https://doi.org/10.1002/pro.3410.

19. Atasoglu C, Valdés C, Walker ND, Newbold CJ, Wallace RJ. De novo synthesis of amino acids by the ruminal bacteria Prevotella bryantii B14, Selenomonas ruminantium HD4, and Streptococcus bovis ES1. Appl Environ Microbiol. 1998;64(8):2836-43.

20. Chang YJ, Pukall R, Saunders E, Lapidus A, Copeland A, Nolan M, Glavina Del Rio T, Lucas S, Chen F, Tice H, Cheng JF, Han C, Detter JC, Bruce D, Goodwin L, Pitluck S, Mikhailova N, Liolios K, Pati A, Ivanova N, Mavromatis K, Chen A, Palaniappan K, Land M, Hauser L, Jeffries CD, Brettin T, Rohde M, Göker M, Bristow J, Eisen JA, Markowitz V, Hugenholtz P, Kyrpides NC, Klenk HP. Complete genome sequence of Acidaminococcus fermentans type strain (VR4). Stand Genomic Sci. 2010;3(1):1-14. https://doi.org/10.4056/sigs. 1002553.

21. Flint HJ, Duncan SH, Scott KP, Louis P. Links between diet, gut microbiota composition and gut metabolism. Proc Nutr Soc. 2015;74:13-22. https://doi. org/10.1017/S0029665114001463.

22. Przybyla-Zawislak B, Dennis RA, Zakharkin SO, McCammon MT. Genes of succinyl-CoA ligase from Saccharomyces cerevisiae. Eur J Biochem. 1998; 258(2):736-43.

23. Schwab C, Gänzle M. Lactic acid bacteria fermentation of human milk oligosaccharide components, human milk oligosaccharides and galactooligosaccharides. FEMS Microbiol Lett. 2011;315:141-8.

24. Frese SA, Parker K, Calvert CC, Mills DA. Diet shapes the gut microbiome of pigs during nursing and weaning. Microbiome. 2015;3:28. https://doi.org/10. 1186/s40168-015-0091-8.

25. Grondin JM, Duan D, Kirlin AC, Abe KT, Chitayat S, Spencer HL, Spencer C, Campigotto A, Houliston S, Arrowsmith $\mathrm{CH}$, Allingham JS, Boraston AB, Smith SP. Diverse modes of galacto-specific carbohydrate recognition by a family 31 glycoside hydrolase from Clostridium perfringens. PLoS One. 2017; 12(2):e0171606. https://doi.org/10.1371/journal.pone.0171606.
26. Zhao R, Zhao R, Tu Y, Zhang X, Deng L, Chen X. A novel a-galactosidase from the thermophilic probiotic Bacillus coagulans with remarkable protease-resistance and high hydrolytic activity. PLoS One. 2018;13(5): e0197067. https://doi.org/10.1371/journal.pone.0197067 eCollection 2018.

27. Kopinski JS, Leibholz J, Love RJ. Biotin studies in pigs. 5. The post-ileal absorption of biotin. Br J Nutr. 1989;62(3):781-9.

28. Asrar FM, O'Connor DL. Bacterially synthesized folate and supplemental folic acid are absorbed across the large intestine of piglets. J Nutr Biochem. 2005;16(10):587-93.

29. Kaiko GE, Stappenbeck TS. Host-microbe interactions shaping the gastrointestinal environment. Trends Immunol. 2014;35(11):538-48. https:// doi.org/10.1016/j.it.2014.08.002.

30. Frosali S, Pagliari D, Gambassi G, Landolfi R, Pandolfi F, Cianci R. How the intricate interaction among toll-like receptors, microbiota, and intestinal immunity can influence gastrointestinal pathology. J Immunol Res. 2015: 489821. https://doi.org/10.1155/2015/489821.

31. Plovier H, Cani PD. Enteroendocrine cells: metabolic relays between microbes and their host. Endocr Dev. 2017;32:139-64. https://doi.org/10. $1159 / 000475736$.

32. Gabler NK, Spurlock ME. Integrating the immune system with the regulation of growth and efficiency. J Anim Sci. 2008;86(14 Suppl):E64-74.

33. Wyns H, Plessers E, De Backer $P$, Meyer E, Croubels S. In vivo porcine lipopolysaccharide inflammation models to study immunomodulation of drugs. Vet Immunol Immunopathol. 2015;166:58-69. https://doi.org/10.1016/ j.vetimm.2015.06.001.

34. Cui W, Chen L, Huang T, Gao Q, Jiang M, Zhang N, Zheng L, Feng K, Cai Y, Wang $\mathrm{H}$. Computationally identifying virulence factors based on KEGG pathways. Mol BioSyst. 2013;9(6):1447-52. https://doi.org/10.1039/ c3mb70024k.

35. National Research Council. Nutrient requirements of swine. 11th ed Washington, DC: National Academy Press; 2012.

36. AOAC. Official methods of analysis. 18th ed. Arlington: Association of Official Analytical Chemists; 2006.

37. Metzler-Zebeli BU, Lawlor PG, Magowan E, Zebeli Q. Effect of freezing conditions on fecal bacterial composition in pigs. Animals (Basel). 2016;6(3). https://doi.org/10.3390/ani6030018.

38. Glass EM, Wilkening J, Wilke A, Antonopoulos D, Meyer F. Using the metagenomics RAST server (MG-RAST) for analyzing shotgun metagenomes. Cold Spring Harb Protoc. 2010;2010(1):pdb-prot5368.

39. Keegan KP, Glass EM, Meyer F. MG-RAST, a metagenomics service for analysis of microbial community structure and function. Methods Mol Biol. 2016;1399:207-33. https://doi.org/10.1007/978-1-4939-3369-3-13.

40. Wilke A, Bischof J, Gerlach W, Glass E, Harrison T, Keegan KP, Paczian T, Trimble WL, Bagchi S, Grama A, Chaterii S, Meyer F. The MG-RAST metagenomics database and portal in 2015. Nucleic Acids Res. 2016;44(D1): D590-4. https://doi.org/10.1093/nar/gkv1322.

41. Rho M, Tang H, Ye Y. FragGeneScan: predicting genes in short and errorprone reads. Nucleic Acids Res. 2010;38:e191. https://doi.org/10.1093/nar/ gkq747

42. Kent WJ. BLAT - the BLAST-like alignment tool. Genome Res. 2002;12:656-4. https://doi.org/10.1101/gr.229202.

43. Kanehisa M, Goto S. KEGG: Kyoto encyclopedia of genes and genomes. Nucleic Acids Res. 2000;28:27-30. https://doi.org/10.1093/Nar/28.1.27.

44. Kanehisa M, Sato Y, Kawashima M, Furumichi M, Tanabe M. KEGG as a reference resource for gene and protein annotation. Nucleic Acids Res. 2016;44:D457-62. https://doi.org/10.1093/nar/gkv1070.

45. Love Ml, Huber W, Anders S. Moderated estimation of fold change and dispersion for RNA-seq data with DESeq2. Genome Biol. 2014;15. https://doi. org/10.1186/s13059-014-0550-8.

46. Benjamini $Y$, Hochberg $Y$. Controlling the false discovery rate: a practical and powerful approach to multiple testing. J R Stat Soc Ser B Methodol. 1995:57:289-300.

47. Oksanen J, Blanchet FG, Friendly M, Kindt R, Legendre P, McGlinn D, Minchin PR, O'Hara R, Simpson GL, Solymos P, Henry M, Stevens H, Szoecs E, Wagner $H$. Vegan: community ecology package. R package version 2.4-3 (date: 2017-04-06). 2017. https://cran.r-project.org/web/packages/vegan/ index.html. 